\title{
Germanium-on-silicon mid-infrared waveguides and Mach-Zehnder interferometers
}

Aditya Malik ${ }^{1,2}$, Muhammad Muneeb ${ }^{1,2,3}$, Yosuke Shimura ${ }^{4,5}$, Joris Van Campenhout ${ }^{4}$,Roger Loo $^{4}$ and Gunther Roelkens ${ }^{1,2,3}$ Email:Aditya.Malik@intec.ugent.be

${ }^{1}$ Photonics Research Group, Ghent University-imec, Sint-Pietersnieuwstraat 41, 9000 Ghent, Belgium,

${ }^{2}$ Center for Nano- and Biophotonics, Ghent University, Belgium,

${ }^{3}$ COBRA Research Institute, Eindhoven University of Technology, P.O. Box 513, Eindhoven 5600 MB, The Netherlands,

${ }^{4}$ imec, Kapeldreef 75, Leuven, 3001, Belgium,

${ }^{5}$ Instituut voor Kern- en Stralingsfysica, KU Leuven, 3001 Leuven, Belgium

\begin{abstract}
In this paper we describe Ge-on-Si waveguides and Mach-Zehnder interferometers operating in the $5.2-5.4 \mu \mathrm{m}$ wavelength range. $3 \mathrm{~dB} / \mathrm{cm}$ waveguide losses and Mach-Zehnder interferometers with $20 \mathrm{~dB}$ extinction ratio are presented.

Index Terms-mid-infrared, photonic integrated circuits, germanium on silicon
\end{abstract}

\section{INTRODUCTION}

The mid-infrared wavelength range is a region of interest for spectroscopic sensing applications, due to strong and specific absorption features of many molecules in that wavelength range [1]. This allows for a non-contact method to detect the presence and concentration of these molecules. Quantum cascade lasers and inter-band cascade lasers are used as coherent laser sources in this wavelength range. These are typically single-wavelength lasers with limited tuning range. Other options are the use of broadband incoherent thermal sources or coherent supercontinuum sources in combination with a spectrometer. Current instrumentation is bulky, expensive and power hungry, necessitating the need for a midinfrared photonic integration platform to mitigate these disadvantages. Several functions can be implemented on such a platform, such as wavelength multiplexers for arrays of single wavelength QCL lasers allowing to cover a broad wavelength range and the integration of spectrometers for spectral analysis of broadband sources after they passed through a gas or liquid under study.

For telecom wavelengths, Silicon-on-Insulator (SOI) is now a standard waveguide platform. One of the main reasons for this is the compatibility with CMOS fabrication technology and the associated potential for large volume manufacturing at low cost. However, beyond $3.8 \mu \mathrm{m}$, the underlying buried oxide layer starts absorbing heavily [2]. There are several candidates for viable waveguide platforms for the mid-IR wavelength range beyond $3.8 \mu \mathrm{m}$ as discussed primarily in [2]. There have been reports in recent literature on $\mathrm{Ge}$-on-Si waveguides [3], free-standing $\mathrm{Si}$ waveguides [4] and silicon-on-sapphire waveguides [5]. In this paper we describe the fabrication of Ge-on-Si photonic integrated circuits, an attractive option for mid-IR photonics and present waveguide losses and a key photonic component in wavelength multiplexers and spectrometers: the Mach-Zehnder Interferometer (MZI).

\section{GERMANIUM-ON-SILICON PLATFORM}

Epitaxial Ge, 1 and $2 \mu \mathrm{m}$ thick was grown on an n-type $\mathrm{Si}(001)$ substrate at imec on $200 \mathrm{~mm}$ wafers by an atmospheric chemical vapor deposition system as described in [6]. Since there is a lattice mismatch of $4.2 \%$ between $\mathrm{Si}$ and $\mathrm{Ge}$, the threading dislocation density(TDD) is quite high at the $\mathrm{Si}-\mathrm{Ge}$ interface. To reduce this threading dislocation density in the film, the Ge is annealed at $850^{\circ} \mathrm{C}$ for 3 minutes. In $1 \mu \mathrm{m}$ thick $\mathrm{Ge}$, the threading dislocation density is $10^{8} \mathrm{~cm}^{-2}$, while for $2 \mu \mathrm{m}$ thick Ge, it is about an order of magnitude less.

To define Ge-on-Si photonic integrated circuits, a Ti/Cr metal mask is used, which is defined using lift-off. i-line contact lithography is used for this purpose. Dry etching of $\mathrm{Ge}$ is then carried out in $\mathrm{CF}_{4} / \mathrm{O}_{2}$ plasma, followed by an $\mathrm{HF}$ dip to remove the metal mask. The waveguides are etched completely through the germanium device layer. A scanning electron microscope picture of such a waveguide structure is shown in Figure 1.

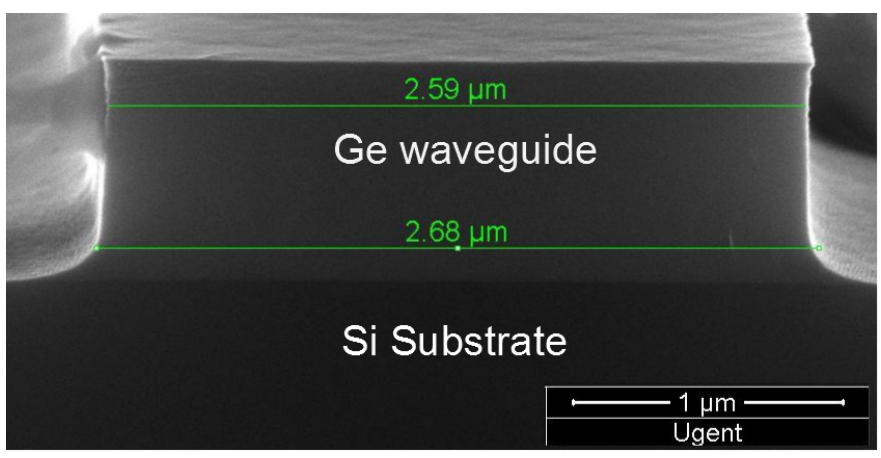

Fig. 1. Ge-on-Si waveguide

\section{MEASUREMENT SET UP}

Light was coupled from a commercially available Daylight Solutions Quantum Cascade Laser in an Indium Fluoride fiber from IRPhotonics using a black diamond aspheric lens. Wire grid polarizers were used to choose the desired polarization in fiber. The cleaved end of the fiber was mounted on a piezo-driven xyz stage to align accurately with the input taper waveguide. Light from the output taper was collected again using a cleaved fiber and then coupled to a InSb detector. To improve the signal to noise ratio, the output of the detector was coupled to a lock-in amplifier. For these measurements the laser was operating at $100 \mathrm{kHz}$ frequency with a duty cycle of $5 \%$ and the trigger from the laser was used as reference for the lock-in amplifier. The laser and lock-in amplifier are addressed by software to scan the wavelength and record the 
corresponding output simultaneously. A schematic diagram of the measurement setup is shown in Figure 2.

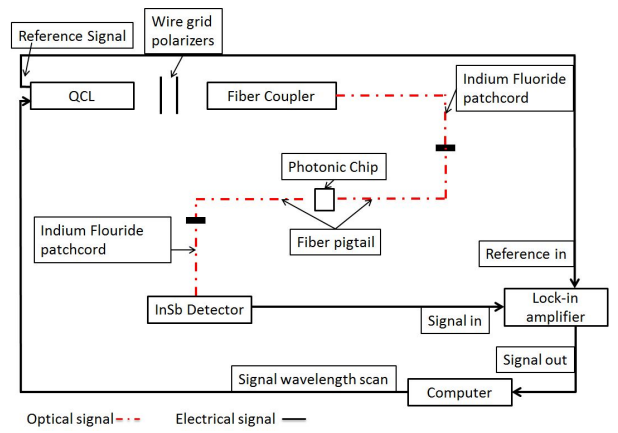

Fig. 2. Measurement Setup

IV. DESIGN AND CHARACTERIZATION OF COMPONENTS

A. Waveguide Losses

Waveguides structures of different lengths on $1 \mu \mathrm{m}$ and $2 \mu \mathrm{m}$ thick germanium-on-silicon wafers were realized. Since the optical mode overlaps less with the Si-Ge interface in $2 \mu \mathrm{m}$ thick Ge and since also the TDD in the bulk of the film is lower, $2 \mu \mathrm{m}$ thick Ge waveguides show lower losses compared to $1 \mu \mathrm{m}$ thick Ge waveguides. The waveguides were connected to a taper for better in and out coupling. Waveguide losses were characterized using a cut back method. The results are shown in Figure 3(a) and 3(b). A waveguide loss for TE polarized light around $3-4 \mathrm{~dB} / \mathrm{cm}$ in the $5.2-5.4 \mu \mathrm{m}$ wavelength range for $2 \mu \mathrm{m}$ thick Ge,which is comparable to the values cited in[3], and 7-8 dB/cm in the 3.7-3.8 $\mu \mathrm{m}$ wavelength range for $1 \mu \mathrm{m}$ thick $\mathrm{Ge}$, is obtained. The waveguide width was 2.25 $\mu \mathrm{m}$ and $2 \mu \mathrm{m}$ respectively to obtain single mode operation.
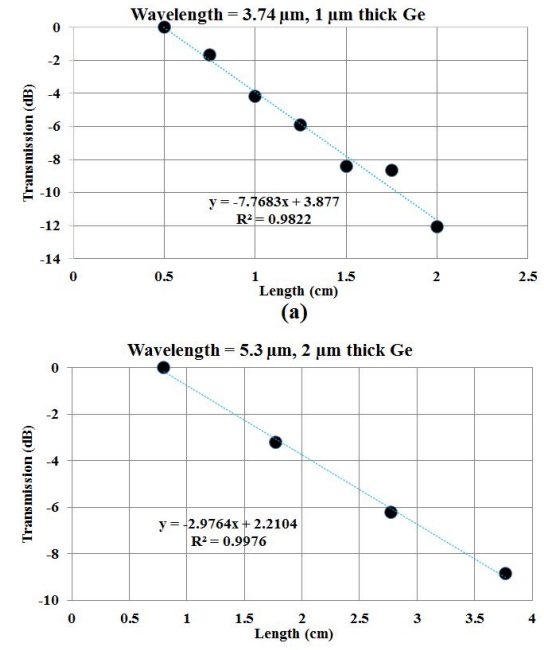

(b)

Fig. 3. Loss measurements for (a) $1 \mu \mathrm{m}$ thick Ge waveguides at $3.74 \mu \mathrm{m}$ wavelength and (b) $2 \mu \mathrm{m}$ thick Ge waveguides at $5.3 \mu \mathrm{m}$ wavelength

\section{B. Mach-Zehnder interferometers}

Since Mach-Zehnder interferometers are key components for wavelength multiplexers and spectrometers, we focus on this component in this paper. The $3 \mathrm{~dB}$ splitters and combiners required for these devices can be implemented in various ways. In this work multimode interferometers (MMIs) are used, given the fact that they are wide-band and do not require sub-micron critical device dimensions. $1 \times 2$ and $2 \times 2$ MMIs were designed for TE polarization using the full-vectorial eigenmode expansion tool FIMMWAVE. For a $25 \mu \mathrm{m}$ wide multimode waveguide region with $10 \mu \mathrm{m}$ wide input and output waveguides a 50:50 splitting was obtained for a device length of $235 \mu \mathrm{m}$ and $930 \mu \mathrm{m}$ for a $1 \times 2$ and $2 \times 2 \mathrm{MMI}$ respectively.

\section{MZI Measurements}

A $1 \times 1$ MMI was combined with $2 \times 2$ MMI to form a $1 \times 2$ MZI with a path length difference of $130 \mu \mathrm{m}$ between both arms. The response of this device is shown in Figure 4. $20 \mathrm{~dB}$ extinction is obtained around $5321 \mathrm{~nm}$, while the insertion loss of the device is within the measurement accuracy of the setup. This illustrates the potential of these devices to be used in wavelength multiplexers for QCL light engines and midinfrared broadband spectrometers.

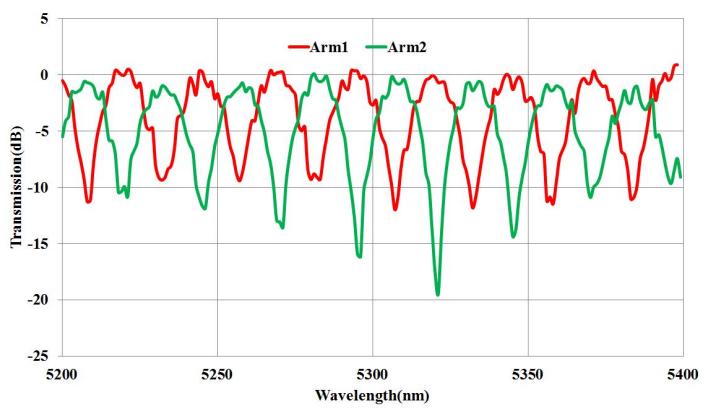

Fig. 4. Response of the realized $1 \times 2 \mathrm{MZI}$

\section{CONClusion}

Ge-on-Si waveguides, MMIs and MZIs with low loss and promising performance in the $5 \mu \mathrm{m}$ wavelength range have been demonstrated for the first time. This provides basic building blocks for more complicated photonic integrated circuits to realize complete on-chip spectroscopic systems.

\section{ACKNOWLEDGMENT}

This work has been carried out in the framework of FP7-ERC project MIRACLE. Yosuke Shimura acknowledges the FWO for a Pegasus Marie Curie Fellowship.

\section{REFERENCES}

[1] R.F.Curl et.al., "Quantum cascade lasers in chemical physics", Chemical Physics Letters 487 (2010) 1-18.

[2] R.A.Soref, S.J.Emelett and W.R.Buchwald,"Silicon waveguide components for the long-wave infrared region"', Journal of Optics A: Pure and Applied Optics, 8(2006), pp. 840-848.

[3] Y.C. Chang et.al., "Low-loss germanium strip waveguides on silicon for the mid-infrared",Optics Letters July 15, 2012, Vol. 37, No. 14, pp. 2883-2885.

[4] Y.Xia et.al., "Suspended Si ring resonator for mid-IR application",Optics Letters, Vol.38,No.7,April 1,2013, pp. 1122-1124.

[5] T.B.Jones et.al.,"Silicon-on-sapphire integrated waveguides for the midinfrared",Optics Express,24 May 2010,Vol.18,No. 12,pp.12127-12135

[6] R.Loo et.al., "High Quality Ge Virtual Substrates on Si Wafers with Standard STI Patterning",Journal of The Electrochemical Society, 157 (1) $H 13-H 21$ (2010). 